\title{
A novel RING finger in the C-terminal domain of the coatomer protein a-COP
}

\author{
Gurmeet Kaur $^{*}$ and Srikrishna Subramanian ${ }^{*}$
}

\begin{abstract}
The C-terminal domain of a-COP, an essential subunit of the COPI coatomer complex, is composed of an all a-helical region and a small $\beta$-sheet domain. We show that this $\beta$-sheet domain is a Really Interesting New Gene (RING)-like treble clef zinc finger. The zinc-binding residues are substituted by other aminoacids in many homologs including the structurally-characterized proteins from Saccharomyces cerevisiae and Bos taurus. This RING-like domain is possibly related to those of other vesicle membrane-associated complexes, such as CORVET, HOPS and SEA, and likely mediates interactions with Dsl1p and assist in coat oligomerization.
\end{abstract}

Keywords: Cellular transport, Coat protein, Coated vesicles, Tethering complex, Zinc finger

\section{Finding}

The cytoplasmic coat protein I (COPI) complex, which coats the vesicles involved in retrograde transport of proteins from the Golgi apparatus to the endoplasmic reticulum (ER), and in intra-Golgi protein trafficking, comprises of a coatomer and ADP-ribosylation factor 1 (ARF) GTPase [1-3]. The coatomer is a heptameric protein complex with two distinct subcomplexes: an inner cargo-binding heterotetrameric F-subcomplex consisting of $\beta-, \gamma^{-}, \delta$ - and $\zeta$-COP, and an outer cage-forming heterotrimeric B-subcomplex consisting of $\alpha$-, $\beta$ '- and $\varepsilon$-COP. $\alpha$ COP subunit is a $\sim 1200$-residue protein with a $\mathrm{N}$-terminal $\beta$-propeller domain ( 600-residues; PDBid 4J8G_A), an $\alpha$ helical region $(\sim 200$-residues; partial structure in PDBid 3MKQ_B), an unstructured region ( 100-residues) and a C-terminal domain (CTD; 300-residues; PDBid 3MKR_B from Bos taurus; PDBid 3MV3_A from Saccharomyces cerevisiae) (Fig. 1a) [4-7]. The CTD of $\alpha$-COP adopts a ' $U$ 'shaped structure, where an initial stack of $11 \alpha$-helices is followed by a $\beta$-sheet domain that constitute the descending and the ascending arm of the ' $U$ ', respectively $[6,7]$.

The $\mathrm{C}$-terminal $\beta$-sheet domain of $\alpha$-COP is necessary for its interaction with- and incorporation of- $\varepsilon$-COP into the coatomer, for maintaining normal levels of $\varepsilon$-COP, and has also been shown to interact with the $\beta$ '-subunit [8]. The yeast ret1-3 allele of $\alpha$-COP (S1188F mutation in the $\beta$ -

\footnotetext{
* Correspondence: gurmeetkaur@imtech.res.in; krishna@imtech.res.in

CSIR-Institute of Microbial Technology (IMTECH), Sector 39-A, Chandigarh 160036, India
}

(C) 2015 Kaur and Subramanian. Open Access This article is distributed under the terms of the Creative Commons Attribution 4.0 International License (http://creativecommons.org/licenses/by/4.0/), which permits unrestricted use, distribution, and reproduction in any medium, provided you give appropriate credit to the original author(s) and the source, provide a link to the Creative Commons license, and indicate if changes were made. The Creative Commons Public Domain Dedication waiver (http://creativecommons.org/publicdomain/zero/1.0/) applies to the data made available in this article, unless otherwise stated.

sheet domain) causes instability of $\alpha$-COP and the coatomer, defects in cellular transport and temperature sensitivity in the organism [8-10]. The CTD has also been suggested to play a role in mediating interactions with Dsl1p subunit of the Dsl1 tethering complex on the ER membranes and assist in the fusion of COPI-coated vesicular membrane with the target membrane $[7,10]$.

Currently, Pfam [11] and Conserved Domain Database (CDD) [12], co-classify the complete stretch of $\alpha$-COP CTD (Pfam: PF06957; CDD: cl18502). We show that the $\beta$-sheet region of $\alpha$-COP CTD (PDBid 3MKR_B, residues 1164-1212; PDBid 3MV3_A, residues 1145-1193) is a Really Interesting New Gene (RING)-like binuclear treble clef zinc finger domain. However, the $\beta$-sheet domain of $\alpha$-COP CTD has undergone structural changes and lost its ability to chelate zinc in many organisms.

A multiple sequence alignment of the $\beta$-sheet domain of representative $\alpha$-COP sequences, obtained after PSIBLAST [13] and JackHMMER [14] sequence-similarity searches (Additional file 1), reveals putative zinc-chelating residues in homologous sequences at expected structural positions of a RING-like domain (Fig. 1b). We observe strong conservation of aminoacids coordinating the second metal ion. Residues coordinating the first metal ion are substituted by other aminoacids in many organisms, except for the first cysteine that is highly conserved (Additional file 2). Of the sequences retrieved, $\alpha$-COP $\beta$-sheet domain would likely bind two metal ions in Trypanosoma cruzi (gi|407411736) and Colletotrichum 


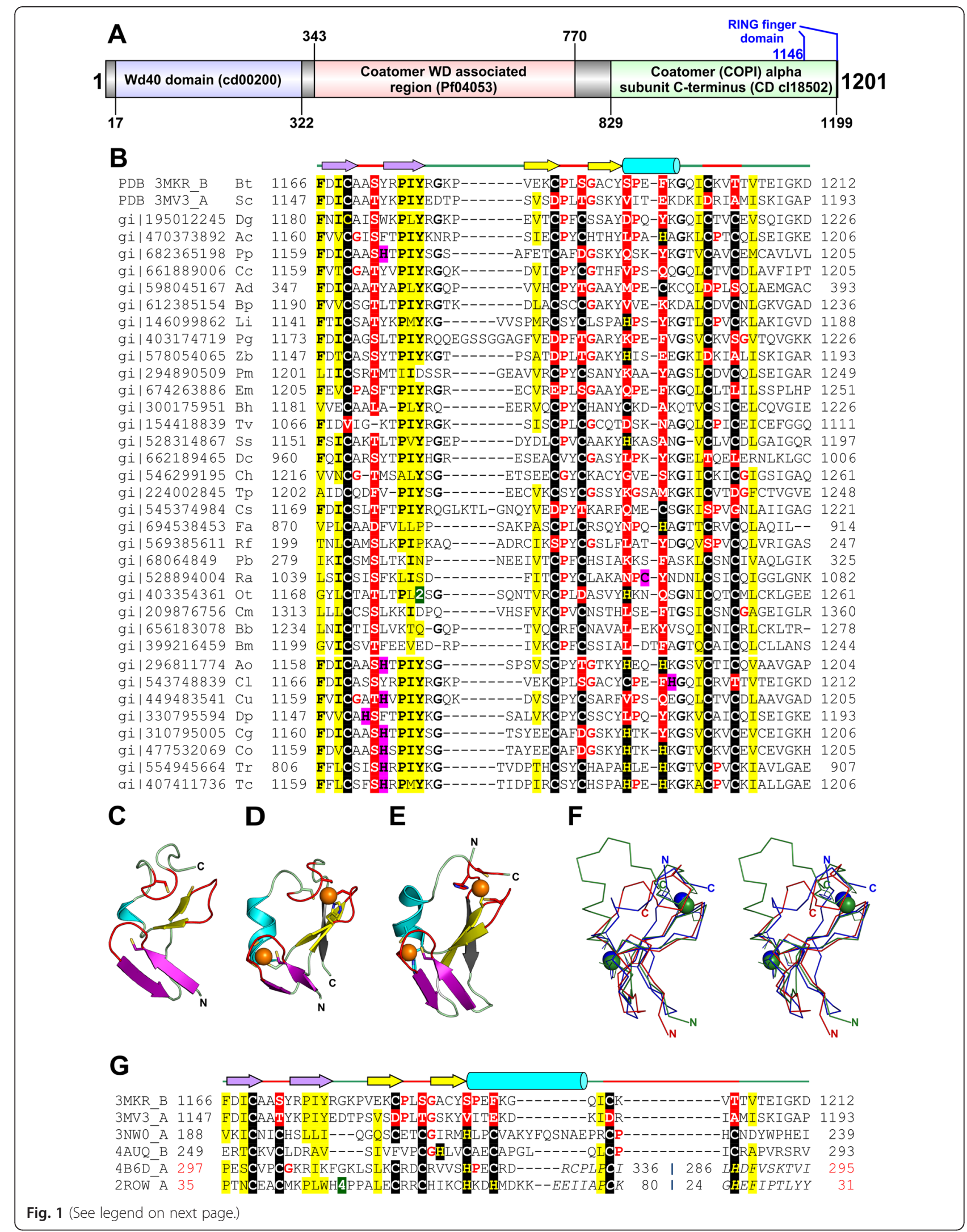


(See figure on previous page.)

Fig. 1 Structure and sequence comparison of a-COP and other RING fingers. a Domain diagram of S. cerevisiae a-COP. Domain boundaries were obtained by referring to CDD (Additional file 1). Position of the manually delineated RING finger domain is indicated in blue. b Structure-based multiple sequence alignment of RING domain from a-COP homologs. c RING domain of a-COP (PDBid 3MKR_B) (d) A classical RING finger domain from Baculoviral IAP repeat-containing protein 7 (PDBid 4AUQ_B) (e) A circularly permuted RING finger from Rac GTPase-activating protein 1 (PDBid 4B6D_A). The RING fingers are colored alike, zinc-binding knuckles: red, primary $\beta$-hairpin: yellow, a-helix: cyan, zinc knuckle containing $\beta$-hairpin: violet, the additional $\beta$-strand: gray, zinc ion: orange sphere. The $\mathrm{N}$ - and $\mathrm{C}$-termini are labeled ' $\mathrm{N}$ ' and ' $\mathrm{C}$ ', respectively. Side chains of metal-chelating residues are shown in stick representation. $\mathbf{f}$ Stereo diagram of the superimposition of the a-COP RING finger (PDBid 3MV3_A; colored red), RING finger domain of Non-SMC element 1 homolog (PDBid 3NWO_B; colored green) and C1 domain of Rac GTPase-activating protein 1 (PDBid 4B6D_A; colored blue). The $C_{a}$ backbone trace is shown for each structure, and bound zinc ions are shown as spheres in the respective colors. $\mathbf{g}$ Structure-based sequence alignment of RING domain of a-COP (PDBid 3MKR_B, 3MV3_A), RING finger domains (PDBid 3NWO_A, 4AUQ_B) and circularly permuted RING fingers (PDBid 4B6D_A, 2ROW_A). For panels $(\mathbf{b}, \mathbf{g})$ PDBid/Gene identification (gi) number, the first and the last residue numbers of the sequences depicted in the alignment are indicated for each sequence. The secondary structure elements of the RING finger domain are indicated above the alignment. The potential metal-binding ligands are boxed in black, non-metal-binding residues at the same position are boxed in red and adjacent residues that could potentially chelate metal ion are boxed in magenta. Conserved residues are in bold. Small aminoacids (Gly, Pro) in the vicinity of the zinc-chelating ligands are colored red. Uncharged residues (all aminoacids except Asp, Glu, Lys and Arg) in mostly hydrophobic sites are highlighted yellow. Some insertions are not shown and the number of omitted residues is specified by numbers boxed in green. The sequence numbers in the region of permutation in panel ( $\mathbf{g}$ ) are shown in red and regions of circular permutation are separated by a 'I' symbol. In panel (b) the PDBid/gi number is followed by organism name abbreviation as follows: Bt- Bos taurus, Sc- Saccharomyces cerevisiae, Dg- Drosophila grimshawi, Ac- Acanthamoeba castellanii str. Neff, Pp- Pseudogymnoascus pannorum VKM F-4516 (FW-969), Cc- Coffea canephora, Ad- Auricularia delicata TFB-10046 SS5, Bp- Bathycoccus prasinos, Li- Leishmania infantum JPCM5, Pg- Puccinia graminis f. sp. tritici CRL 75-36-700-3, Zb- Zygosaccharomyces bailii ISA1307, Pm- Perkinsus marinus ATCC 50983, Em-Echinococcus multilocularis, Bh- Blastocystis hominis, Tv- Trichomonas vaginalis G3, Ss- Schizosaccharomyces cryophilus OY26, Dc- Diaphorina citri, Ch- Chondrus crispus, Tp- Thalassiosira pseudonana CCMP1335, Cs- Coccomyxa subellipsoidea C-169, Fa- Fonticula alba, Rf- Reticulomyxa filose, Pb- Plasmodium berghei ANKA, Ra- Rozella allomycis CSF55, Ot- Oxytricha trifallax, Cm- Cryptosporidium muris RN66, Bb- Babesia bigemina, Bm- Babesia microti strain RI, Ao- Arthroderma otae CBS 113480, Cl-Columba livia, Cu-Cucumis sativus, Dp- Dictyostelium purpureum, Cg-Colletotrichum graminicola M1.001, Co- Colletotrichum orbiculare MAFF 240422, Tr- Trypanosoma rangeli SC58, TC- Trypanosoma cruzi marinkellei

orbiculare (gi|477532069) (Fig. 1b). Other conserved hydrophobic and aromatic residues are present in the $\beta$ strands and the extended loop region of the domain.

Consistent with the observed pattern of conservation for the zinc-coordinating residues in the $\alpha$-COP $\beta$-sheet domain, which is particularly high for the second metalcoordinating site, sequence-profile-similarity-based searches using HHpred [15] and FFAS [16] find fortuitous matches to many zinc ribbon domains (for example, HHpred, finds TFIIE $\alpha$ zinc ribbon, PDBid 1VD4_A, E-value =0.014). These zinc ribbons are not homologous to the RING domain of $\alpha-C O P$ and lack sequence similarity outside of the metal-coordinating sites. These searches also find matches to RING domains, although with lower statistical significance. HHpred finds matches to Pfam Prokaryotic RING finger family 1 (PF14446, E-value $=0.046)$, and FFAS finds Non-structural maintenance of chromosomes (SMC) element 1 homolog (PDBid 2CT0_A, Score $=-5.28$ ), putative zinc-finger of transcription factor IIIC complex (PF12660, Score $=-5.82)$ and Rho-associated protein kinase 2 (PDBid 2 ROW_A, Score $=-7.64$ ).

Structurally, the $\beta$-sheet domain of $\alpha$-COP consists of two $\beta$-hairpins, a small $\alpha$-helical turn, and a loop with a tight turn (Fig. 1c). Albeit minor structural variations, this topological arrangement resembles a RING-like fold (Fig. 1d) [17-20]. Classical (mononuclear) treble clefs comprise of a zinc knuckle (a non-canonical turn with the sequence CPXCG), a primary $\beta$-hairpin and an $\alpha$-helix, with the zinc-binding site formed by the zinc knuckle and
$\mathrm{N}$-terminal of the $\alpha$-helix [17]. In the RING-like crossbraced binuclear treble clefs, a second zinc ion is coordinated by ligands contributed by the turn of the primary $\beta$-hairpin and an extended knuckle-like region (also referred to as a 'squiggle') after the $\alpha$-helix (Fig. 1d, e) $[18,19]$. Most RING fingers have an additional C-terminal $\beta$-strand that forms a three-stranded $\beta$-sheet with the primary $\beta$-hairpin (Fig. 1d). The second metal-binding site is structurally similar to rubredoxin-like zinc ribbons $[17,21]$.

In the $\beta$-sheet domain from structurally-characterized $\alpha$-COP, the canonical $\alpha$-helix of the treble clef is reduced to a single turn, and the additional $\beta$-strand seen in RING fingers is absent. None-the-less, automated structuresimilarity searches with the $\beta$-sheet domain of $\alpha$-COP using Dali [22] retrieved matches to other treble clefs, with top-scoring matches to RING fingers. The retrieved matches included RING domain of E3 ubiquitin-protein ligase EL5 (PDBid 1IYM_A, Z-score = 3.7, RMSD = 2.4 $\AA$, nali $=42$ ), U-box domain of pre-mRNA splicing factor PRP19 (PDBid 2BAY_D, Z-score $=3.4, \mathrm{RMSD}=2.0 \AA$, nali $=40)$ and $C 1$ domain of Rac GTPase-Activating Protein 1 (PDBid 4B6D_A, Z-score $=3.8, \mathrm{RMSD}=2.4 \AA$, nali $=40)$ (Fig. 1e). We could manually superimpose the $\beta$-sheet domain of $\alpha$-COP (PDBid 3MV3_A) and the RING finger of Non-SMC element 1 homolog (PDBid 3NWO_B) with an RMSD of $1.3 \AA$ over 35 pairs of backbone $\mathrm{C}_{\alpha}$ atoms (Fig. 1f, g). Automated pairwise protein structure superimposition using TM-align [23] and Fr- 
TM-align [24] produced similar results with a TM-score $>0.5$, indicating a fold level similarity of $\alpha$-COP $\beta$-sheet domain with other RING domains.

Zinc finger domains occur in several proteins of cellular transport pathways in a variety of functional contexts (Additional file 2). Interestingly, we find that subunit proteins of few other vesicle membrane-associated complexes such as Vps8, Vps11, Vps18 and Vps39 of the CORVET (class $\mathrm{C}$ core vacuole/endosome tethering) and the late endosomal/lysosomal HOPS (homotypic fusion and protein sorting) complexes [25], and Sea4 of the SEA (Seh1associated) complex [26] share similar domain architectures, including the $\beta$-propeller, the $\alpha$-solenoid and the RING finger, with the $\alpha$-COP. Additionally, Sea2 and Sea3 of the SEA complex also have a RING finger and a $\beta$ propeller but lack the $\alpha$-solenoid region [26]. All the membrane-associated coating- and trafficking-related and nuclear pore complexes are suggested to have diverged from a progenitor 'protocotomer' which was present in the first common eukaryotic ancestor (FECA) [27, 28]. Although our sequence-similarity searches with the RING domain of $\alpha$-COP were not able to retrieve the RING domains of these proteins, based on common domain architectures and similar biological pathways where they function, the RING domains in all these proteins plausibly share a distant evolutionary relationship. The RING domain in the proteins of CORVET and HOPS is important for endolysosomal transport by mediating fusion and docking of vesicles at vacuoles, mediating interaction with other proteins and heterodimerization [29-31]. The RING domain of $\mathrm{Vps} 18$ also mediates ubiquitination in vitro $[32,33]$.

Currently, no specific function is attributed to the RING finger of $\alpha$-COP. It is plausible that like bonafide RING domains, the RING finger of $\alpha$-COP may also possess E3ubiquitin ligase activity that might affect the assembly and function of the coatomer as proposed for SEA-complex proteins [26]. The $\mathrm{C}$-terminal 19 residues of $\alpha$-COP, covering a part of the RING domain, are known to enhance its interaction with Dsl1p [10]. Mediating protein-protein interactions being one of the primary functional attributes of RING fingers [19, 34], this domain in $\alpha$-COP could likely play a role in interacting with Dsl1p to effectuate the fusion of the COPI-coated vesicles with the ER membrane.

The C-terminal 19 residues of $\alpha$-COP have also been suggested to be involved in dimerization and oligomerization when not interacting with Dsl1p [10]. Moreover, the specific regions of the various coatomer subunits that mediate oligomerization during COPI cage formation are not precisely known $[6,35,36]$. Given the known role of RING domains in homodimerization and macromolecular assemblages in various contexts [20], we speculate that the RING finger of $\alpha$-COP could assist in coat oligomerization.
Indeed, a recent study reporting the cryo-electron microscope structure of the COPI coat (PDBid 5A1V) also suggests that the CTD of $\alpha$-COP is one of the flexible connecting domains involved in oligomerization of coat units (triads) [37]. In conclusion, the detection of a novel RING finger in the COPI complex may aid in gaining additional insights into the function and mechanism of the COPI-mediated vesicle transport system.

\section{Response to reviewer and editor-in-chief's comments on the previous version of the manuscript \\ Reviewer 1}

Dr. Ramanathan Sowdhamini

Report form: Kaur and Subramanian report the presence of a RING finger domain in the C-terminal domain of coatomer protein, $\alpha$-COP, after a detailed sequence and structural analyses. $\alpha$-COP forms one of the subunits in the heptameric assembly of cytoplasmic coat protein I. This assembly is present on Golgi apparatus and is responsible for Golgi-to-endoplasmic-reticulum trafficking. Previous reports on structure determination by other groups have mainly concentrated on the quaternary arrangements, interprotomer interactions and overall domain architectures of individual subunits. The putative connections of the $\mathrm{C}$-terminal region of $\alpha$-COP, discussed through sequence and structural investigations, are compelling. Indeed, such RING fingers have already been reported in analogous biological assemblies like vacuole/endosome tethering and homotypic fusion-protein sorting complexes, thereby providing a strong evolutionary backup for this argument. For all these reasons, I would recommend acceptance of the paper. However, I would like to suggest few changes in the presentation of the work and results before it is ready for publication. These six points are covered below.

1. line 53: I think the authors mean 3MKR, not 3MRK. Response: The PDBid has been corrected.

2. line 89: The premise in which the connections were realized needs to argued out well and subsequently it needs to be referred as 'RING domain in the C-terminal region of $\alpha$-COP'. Currently, already there is a mention that the RING finger domain of $\alpha$-COP is the query. Rather, a better argument is to say that when the C-terminal domain of $\alpha-C O P$ was used as a query and aligned with homologues, quite unexpectedly, several homologues were observed to contain a zinc-finger domain. In particular, you may also point out homologues which are annotated to contain a RING-finger domain.

Response: The suggested change has been included in the present version of the manuscript. None of the $\alpha-C O P$ homologs retrieved during our analysis were previously annotated to contain a RING finger domain. 
3. line 135: Do these searches first pick up zinc ribbons and then ring fingers? If so, it will be nice to mention score for zinc ribbons as well.

Response: We have included the scores for one of the zinc ribbon domain retrieved in the HHpred search.

4. line 170: The rigid-body superposition needs to be discussed before the visual and more subjective comparisons.

Response: We have discussed the Dali results before the manual structure analysis.

5. line 247: "This RING finger has lost its ability to bind to zinc ions ..." This phrase appears too speculative since there are atypical zinc-chelating residues (like 3His-Asp as in adiponectin receptors (http://www.nature.com/nature/ journal/v520/n7547/fig_tab/nature14301_SF4.html), where a water molecule participates to stabilize zinc binding). There could also be compensatory chelating sites owing to oligomerization. Hence, it may be better to state "This RING finger has three of the four classical Zn-binding residues mutated' or 'Sequence analysis suggests that this RING finger may not have zinc-binding function ...'

Response: These lines have been edited in the revised manuscript.

6. line 276: Specific reference to PYMOL visualization maybe avoided.

Response: We have retained the reference to PyMOL in the methods section in the Additional file 1 as we used it extensively for doing manual structure analysis.

Dr. Eugene Koonin

The finding that you report clearly is valid and of certain importance. At the same time, it is highly specific, so I think you made the right choice of describing your finding in a Discovery Note. However, the present manuscript is far too long and detailed for this format, and this could, I believe, discourage Editorial Board members from handling the submission. My suggestion is that, if you remain interested in Biology Direct as the publication venue, you substantially shorten the manuscript by dropping nonessential details and references.

Response: We have edited the manuscript to suit the submission criteria for a Discovery Note.

\section{Additional files}

Additional file 1: Methods. Methods employed in this study (DOCX $32 \mathrm{~kb}$ )

Additional file 2: Supplementary results. Additional supportive evidence in favor of the results presented (DOCX $31 \mathrm{~kb}$ )

\section{Abbreviations}

COP: Cytoplasmic coat protein; RING: Really Interesting New Gene; CORVET: Class C core vacuole/endosome tethering; HOPS: Homotypic fusion and protein sorting; SEA: Seh1-associated; ER: Endoplasmic reticulum; ARF: ADP-ribosylation factor; CTD: C-terminal domain; CDD: Conserved domain database; SMC: Non-structural maintenance of chromosomes; FECA: first common eukaryotic ancestor; PDB: Protein Data Bank.

\section{Competing interests}

The authors declare that they have no competing interests.

\section{Authors' contributions}

GK identified the RING domain in a-COP. GK and SS discussed the relevance of the find and wrote the manuscript. Both authors read and approved the manuscript.

\section{Acknowledgments}

This work was supported in part by the XII Five-year plan network project GENESIS (BSC0121) and intramural funds (OLP_0072) from the Council of Scientific and Industrial Research (CSIR) - Institute of Microbial Technology, Chandigarh, India. G.K. is supported by the Shyama Prasad Mukherjee Fellowship of CSIR, India.

Received: 24 November 2015 Accepted: 3 December 2015 Published online: 14 December 2015

\section{References}

1. Kirchhausen T. Three ways to make a vesicle. Nat Rev Mol Cell Biol. 2000; 1(3):187-98. doi:10.1038/35043117.

2. Faini M, Beck R, Wieland FT, Briggs JA. Vesicle coats: structure, function, and general principles of assembly. Trends Cell Biol. 2013;23(6):279-88. doi:10.1016/j.tcb.2013.01.005.

3. Waters MG, Serafini T, Rothman JE. 'Coatomer': a cytosolic protein complex containing subunits of non-clathrin-coated Golgi transport vesicles. Nature. 1991;349(6306):248-51. doi:10.1038/349248a0.

4. Gerich B, Orci L, Tschochner H, Lottspeich F, Ravazzola M, Amherdt M, et al. Non-clathrin-coat protein alpha is a conserved subunit of coatomer and in Saccharomyces cerevisiae is essential for growth. Proc Natl Acad Sci U S A. 1995;92(8):3229-33.

5. Ma W, Goldberg J. Rules for the recognition of dilysine retrieval motifs by coatomer. EMBO J. 2013;32(7):926-37. doi:10.1038/emboj.2013.41.

6. Lee C, Goldberg J. Structure of coatomer cage proteins and the relationship among COPI, COPII, and clathrin vesicle coats. Cell. 2010;142(1):123-32. doi:10.1016/j.cell.2010.05.030.

7. Hsia KC, Hoelz A. Crystal structure of alpha-COP in complex with epsilon-COP provides insight into the architecture of the COPI vesicular coat. Proc Natl Acad Sci U S A. 2010;107(25):11271-6. doi:10.1073/pnas.1006297107.

8. Eugster A, Frigerio G, Dale M, Duden R. COP I domains required for coatomer integrity, and novel interactions with ARF and ARF-GAP. EMBO J. 2000;19(15):3905-17. doi:10.1093/emboj/19.15.3905.

9. Duden R, Kajikawa L, Wuestehube L, Schekman R. epsilon-COP is a structural component of coatomer that functions to stabilize alpha-COP. EMBO J. 1998;17(4):985-95. doi:10.1093/emboj/17.4.985.

10. Zink S, Wenzel D, Wurm CA, Schmitt HD. A link between ER tethering and COP-I vesicle uncoating. Dev Cell. 2009;17(3):403-16. doi:10.1016/j.devcel.2009.07.012.

11. Punta M, Coggill PC, Eberhardt RY, Mistry J, Tate J, Boursnell C, et al. The Pfam protein families database. Nucleic Acids Res. 2012;40(Database issue):29.

12. Marchler-Bauer A, Zheng C, Chitsaz F, Derbyshire MK, Geer LY, Geer RC, et al. CDD: conserved domains and protein three-dimensional structure. Nucleic Acids Res. 2013;41(Database issue):D348-52. doi:10.1093/nar/gks1243.

13. Altschul SF, Madden TL, Schaffer AA, Zhang J, Zhang Z, Miller W, et al. Gapped BLAST and PSI-BLAST: a new generation of protein database search programs. Nucleic Acids Res. 1997;25(17):3389-402.

14. Finn RD, Clements J, Eddy SR. HMMER web server: interactive sequence similarity searching. Nucleic Acids Res. 2011;39(Web Server issue):18.

15. Soding J, Biegert A, Lupas AN. The HHpred interactive server for protein homology detection and structure prediction. Nucleic Acids Res. 2005; 33(Web Server issue):W244-8. doi:10.1093/nar/gki408.

16. Jaroszewski L, Rychlewski L, Li Z, Li W, Godzik A. FFAS03: a server for profile-profile sequence alignments. Nucleic Acids Res. 2005;33(Web Server issue):W284-8. doi:10.1093/nar/gki418.

17. Grishin NV. Treble clef finger-a functionally diverse zinc-binding structural motif. Nucleic Acids Res. 2001;29(8):1703-14.

18. Krishna SS, Majumdar I, Grishin NV. Structural classification of zinc fingers: survey and summary. Nucleic Acids Res. 2003;31(2):532-50.

19. Burroughs AM, lyer LM, Aravind L. Functional diversification of the RING finger and other binuclear treble clef domains in prokaryotes and the early evolution of the ubiquitin system. Mol BioSyst. 2011;7(7):2261-77. doi:10.1039/c1mb05061c 
20. Borden KL. RING domains: master builders of molecular scaffolds? J Mol Biol. 2000;295(5):1103-12. doi:10.1006/jmbi.1999.3429.

21. Kaur G, Subramanian S. The UBR-box and its relationship to binuclear RING-like treble clef zinc fingers. Biol Direct. 2015;10(1):36. doi:10.1186/s13062-015-0066-5.

22. Holm L, Sander C. Dali: a network tool for protein structure comparison. Trends Biochem Sci. 1995;20(11):478-80.

23. Zhang Y, Skolnick J. TM-align: a protein structure alignment algorithm based on the TM-score. Nucleic Acids Res. 2005;33(7):2302-9. doi:10.1093/nar/gki524

24. Pandit SB, Skolnick J. Fr-TM-align: a new protein structural alignment method based on fragment alignments and the TM-score. BMC Bioinformatics. 2008:9:531. doi:10.1186/1471-2105-9-531.

25. Nickerson DP, Brett CL, Merz AJ. Vps-C complexes: gatekeepers of endolysosomal traffic. Curr Opin Cell Biol. 2009;21(4):543-51. doi:10.1016/j.ceb.2009.05.007.

26. Dokudovskaya S, Waharte F, Schlessinger A, Pieper U, Devos DP, Cristea IM et al. A conserved coatomer-related complex containing Sec13 and Seh1 dynamically associates with the vacuole in Saccharomyces cerevisiae. MCP. 2011;10(6):M110 006478. doi:10.1074/mcp.M110.006478.

27. Field MC, Sali A, Rout MP. Evolution: On a bender-BARs, ESCRTs, COPs, and finally getting your coat. J Cell Biol. 2011;193(6):963-72. doi:10.1083/jcb.201102042.

28. Devos D, Dokudovskaya S, Alber F, Williams R, Chait BT, Sali A, et al. Components of coated vesicles and nuclear pore complexes share a common molecular architecture. PLoS Biol. 2004;2(12), e380. doi:10.1371/journal.pbio.0020380.

29. Plemel RL, Lobingier BT, Brett $C L$, Angers CG, Nickerson DP, Paulsel A, et al. Subunit organization and Rab interactions of Vps-C protein complexes that control endolysosomal membrane traffic. Mol Biol Cell. 2011;22(8):1353-63. doi:10.1091/mbc.E10-03-0260.

30. Rieder SE, Emr SD. A novel RING finger protein complex essential for a late step in protein transport to the yeast vacuole. Mol Biol Cell. 1997;8(11): 2307-27.

31. Horazdovsky BF, Cowles CR, Mustol P, Holmes M, Emr SD. A novel RING finger protein, Vps8p, functionally interacts with the small GTPase, Vps21p, to facilitate soluble vacuolar protein localization. J Biol Chem. 1996:271(52): 33607-15.

32. Yogosawa S, Hatakeyama S, Nakayama Kl, Miyoshi H, Kohsaka S, Akazawa C Ubiquitylation and degradation of serum-inducible kinase by hVPS18, a RING-H2 type ubiquitin ligase. J Biol Chem. 2005;280(50):41619-27. doi:10.1074/jbc.M508397200.

33. Yogosawa S, Kawasaki M, Wakatsuki S, Kominami E, Shiba Y, Nakayama K, et al. Monoubiquitylation of GGA3 by hVPS18 regulates its ubiquitin-binding ability. Biochem Biophys Res Commun. 2006;350(1):82-90. doi:10.1016/j.bbrc.2006.09.013

34. Borden KL. RING fingers and B-boxes: zinc-binding protein-protein interaction domains. Biochemistry and cell biology $=$. Biochimie et biologie cellulaire. 1998;76(2-3):351-8

35. Faini M, Prinz S, Beck R, Schorb M, Riches JD, Bacia K, et al. The structures of $\mathrm{COPI}$-coated vesicles reveal alternate coatomer conformations and interactions. Science. 2012;336(6087):1451-4. doi:10.1126/science.1221443.

36. Jackson LP. Structure and mechanism of COPI vesicle biogenesis. Curr Opin Cell Biol. 2014;29:67-73. doi:10.1016/j.ceb.2014.04.009

37. Dodonova SO, Diestelkoetter-Bachert P, von Appen A, Hagen WJ, Beck R Beck $M$, et al. VESICULAR TRANSPORT. A structure of the COPI coat and the role of coat proteins in membrane vesicle assembly. Science. 2015 349(6244):195-8. doi:10.1126/science.aab1121.

\section{Submit your next manuscript to BioMed Central and we will help you at every step:}

- We accept pre-submission inquiries

- Our selector tool helps you to find the most relevant journal

- We provide round the clock customer support

- Convenient online submission

- Thorough peer review

- Inclusion in PubMed and all major indexing services

- Maximum visibility for your research

Submit your manuscript at www biomedcentral com/submit 